\title{
Robustness of a high-resolution central scheme for hydrodynamic simulations in full general relativity
}

\author{
Masaru Shibata ${ }^{1}$ and José A. Font ${ }^{2}$ \\ ${ }^{1}$ Graduate School of Arts and Sciences, University of Tokyo, Komaba, Meguro, Tokyo 153-8902, Japan \\ ${ }^{2}$ Departamento de Astronomía y Astrofísica, Universidad de Valencia, 46100 Burjassot (Valencia), Spain
}

\begin{abstract}
A recent paper by Lucas-Serrano et al. [1] indicates that a high-resolution central (HRC) scheme is robust enough to yield accurate hydrodynamical simulations of special relativistic flows in the presence of ultrarelativistic speeds and strong shock waves. In this paper we apply this scheme in full general relativity (involving dynamical spacetimes), and assess its suitability by performing test simulations for oscillations of rapidly rotating neutron stars and merger of binary neutron stars. It is demonstrated that this HRC scheme can yield results as accurate as those by the socalled high-resolution shock-capturing (HRSC) schemes based upon Riemann solvers. Furthermore, the adopted HRC scheme has increased computational efficiency as it avoids the costly solution of Riemann problems and has practical advantages in the modeling of neutron star spacetimes. Namely, it allows simulations with stiff equations of state by successfully dealing with very lowdensity unphysical atmospheres. These facts not only suggest that such a HRC scheme may be a desirable tool for hydrodynamical simulations in general relativity, but also open the possibility to perform accurate magnetohydrodynamical simulations in curved dynamic spacetimes.
\end{abstract}

04.25.Dm, 04.30.-w, 04.40.Dg

Hydrodynamics simulation in general relativity (GR) is the best theoretical approach for investigating dynamical phenomena in relativistic astrophysics such as stellar core collapse to a neutron star and a black hole, and the merger of binary neutron stars. In the past several years this field has witnessed major development, to the stage that it is now feasible to perform accurate simulations for such general relativistic phenomena (see e.g. [2-6]). Currently, the most favored approach to hydrodynamics simulations in full GR combines the use of the so-called BSSN formalism to solve Einstein's field equations [7] and upwind high-resolution shock-capturing (HRSC) schemes to solve the hydrodynamics equations [8] in conservation form. Hereafter, HRSC schemes are referred to as those in which the hydrodynamics equations are solved by means of (either exact or approximate) Riemann solvers $[9,8]$ (i.e. Godunov-type schemes).

Regarding the solution of the hydrodynamics equations it has been shown in a few recent papers $[10,1]$ that highresolution central symmetric schemes (HRC scheme hereafter) yield numerical solutions as accurate as those by HRSC schemes for special relativistic flows (see e.g. [11] for a general introduction to HRSC and HRC schemes). The main conclusion of those works highlights the importance of the conservation form of the adopted scheme (either upwind or central) in conjunction with high-order cell-reconstruction procedures (to compute the numerical hydrodynamical fluxes at cell interfaces) to gain accuracy while reducing as much as possible the inherent diffusion of central schemes at discontinuities. It is wellknown that if a numerical scheme written in conserva- tion form converges, it automatically guarantees the correct Rankine-Hugoniot (jump) conditions across discontinuities. This shock-capturing property is hence shared by both upwind and symmetric schemes. For practical reasons the most appealing feature of HRC schemes is the fact that, contrary to upwind HRSC schemes, they entirely sidestep the use of Riemann solvers, which results in a great simplification for their numerical implementation as well as in enhanced computational efficiency. However, it has not yet been clarified whether HRC schemes can also yield numerical results as accurate as those of HRSC schemes for simulations in full GR involving dynamical spacetimes.

The aim of this paper is to demonstrate the robustness of a particular HRC scheme proposed by [12], and first used in special relativistic hydrodynamics by [1], for problems in full GR. As we have done in previous papers (e.g. $[2,3,13])$, test simulations in both axisymmetry (rotating neutron stars) and full three-dimension (binary neutron star mergers) are performed to assess this fact.

The numerical simulations are carried out using the same mathematical formulation as in [5], to which the interested reader is addressed for details about the basic equations, the gauge conditions, and the computational method. Einstein's evolution equations are solved using the so-called BSSN formalism [7], adopting a slight variation of the original form of the equations, which is reported in [5]. The hydrodynamics equations are written in conservation form and solved using both a Roe-type HRSC scheme [13] and a HRC scheme [1], with either the PPM third-order cell-reconstruction or the MC slope 
limiter. Violations of the Hamiltonian constraint and conservation of ADM mass and angular momentum are monitored to check the accuracy of the simulations.

We use a fixed uniform grid for both the axisymmetric and the three-dimensional (3D) simulations. The former are carried out in cylindrical coordinates $(\varpi, z)$ assuming equatorial plane symmetry. Computational grids of size $(N+1, N+1)$ with $N=90,120,180,240$, and 360 are used, with which convergence is shown. The $3 \mathrm{D}$ simulations are performed in Cartesian coordinates assuming equatorial plane symmetry as well. In this case the grid adopted in the present test simulations consists of $(377,377,189)$ zones for $(x, y, z)$ respectively.

In the axisymmetric simulations of isolated rotating neutron stars a $\Gamma$-law equation of state (EOS) is used, i.e. $P=(\Gamma-1) \rho \varepsilon$. Here, $P$ is the pressure, $\rho$ the restmass density, $\varepsilon$ the specific internal energy, and $\Gamma$ the adiabatic constant for which we choose the values 2 and 2.5. The initial conditions for the equilibrium models are built using a polytropic $\operatorname{EOS} P=K \rho^{\Gamma}$, where $K$ is the polytropic constant.

Correspondingly, for the 3D simulations of binary neutron star merger a hybrid EOS is adopted, as described in [6]. In this EOS, the pressure and the specific internal energy are written in the form $P=P_{\text {cold }}(\rho)+P_{\text {th }}$ and $\varepsilon=\varepsilon_{\text {cold }}(\rho)+\varepsilon_{\text {th }}$ where $P_{\text {cold }}$ and $\varepsilon_{\text {cold }}$ are the cold (zero-temperature) parts, and are functions of $\rho$ only. On the other hand $P_{\text {th }}$ and $\varepsilon_{\text {th }}$ are the thermal (finitetemperature) parts. During the simulation, $\rho$ and $\varepsilon$ are evolved, and thus $\varepsilon_{\text {th }}$ is determined by $\varepsilon-\varepsilon_{\text {cold }}$. For $P_{\text {th }}$, we simply set $P_{\text {th }}=\left(\Gamma_{\text {th }}-1\right) \rho \varepsilon_{\text {th }}$ with $\Gamma_{\text {th }}=2$. For the cold part of the hybrid EOS we use realistic EOS for zero-temperature nuclear matter, more precisely the SLy EOS [14].

As customary in grid-based hydrodynamics codes an artificial low-density atmosphere needs to be used in those regions outside the star representing vacuum. The density has to be low enough so that its presence does not affect the actual dynamics of the star. In previous simulations using a Roe-type HRSC scheme, a uniform density atmosphere as low as $\rho_{\text {atm }}=10^{-6} \rho_{\text {max }}$ was used, where $\rho_{\max }$ is the maximum density. (For soft EOS this value can be much smaller; e.g., $\rho_{\text {atm }} \leq 10^{-12} \rho_{\max }$ for $\Gamma=4 / 3$.) Lower values for the density could result in numerical instabilities developing around the stellar surface. However, we have found that when using the HRC scheme the threshold density in the atmosphere can be much smaller. The results presented next for the HRC scheme correspond to $\rho_{\mathrm{atm}}=10^{-10} \rho_{\max }$, irrespective of the EOS used.

We start discussing axisymmetric simulations of oscillations of rotating neutron stars. For these simulations we build rapidly rotating neutron stars with uniform angular velocity. This velocity is chosen so that it reaches the Kepler (mass-shedding) limit at the equatorial stellar surface.

Two rotating neutron star models are considered. In one case $\Gamma=2$ and the baryon rest mass $M_{*}$ is $90 \%$ of the

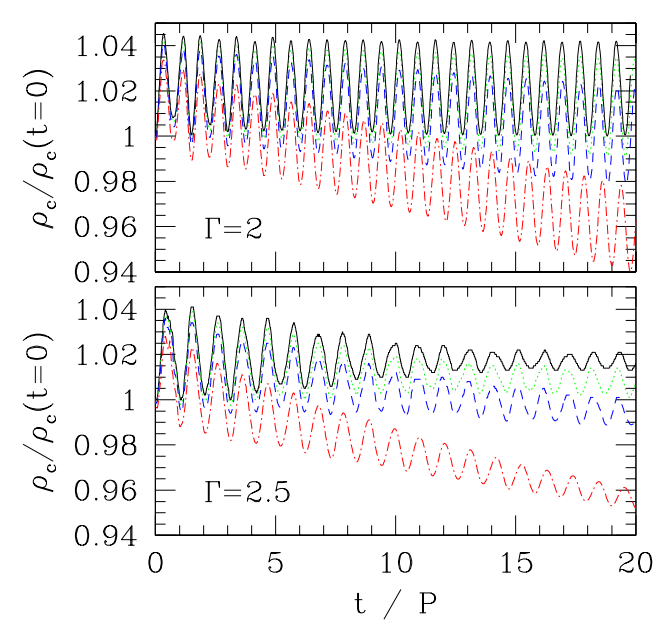

FIG. 1. Evolution of the central density in units of the initial value for the two rotating neutron star models considered. Time is shown in units of the rotational period of the neutron stars $P$. The dotted-dashed, dashed, dotted, and solid curves denote the results with $(121,121),(181,181),(241,241)$, and $(361,361)$ grid resolutions, respectively.

maximum allowed value for uniformly rotating neutron stars of identical EOS. This model is the same as model R2 in Ref. [13], which allows for a direct comparison. The other model corresponds to $\Gamma=2.5$ and $M_{*}$ is $95 \%$ of the maximum allowed mass. This is a very compact model, since the compactness parameter, defined as $G M / R c^{2}$ where $M$ and $R$ are the ADM mass and circumferential radius around the equatorial surface, is 0.214 . For both models, the axis ratio of polar radius to equatorial radius is about 0.6. The ratio of the coordinate radius of the outer boundary of the computational grid to the stellar coordinate radius at the equator is 3 . The simulations are started by reducing the pressure by $1 \%$ uniformly.

Figure 1 shows the time-evolution of the central density for these two models obtained using the HRC scheme for the hydrodynamics equations. Each curve corresponds to a different grid resolution as explained in the caption. It is found that the HRC scheme succeeds in keeping the stars in equilibrium in such a dynamical spacetime. The neutron star oscillations can be followed accurately for more than 20 rotation periods. With small grid sizes (dotted and dashed lines), the density experiences a secular drift, decreasing with time gradually. The reason is that the angular momentum of the star is transported outward by numerical diffusion. However, this drift decreases with improved grid resolution, and with the highest resolution the average value of the central density is kept approximately constant. Second-order convergence is also achieved.

It is worth to emphasize that despite the use of an artificial atmosphere of tiny density, the HRC scheme makes it possible to follow the evolution of compact neutron stars with stiff EOS with $\Gamma=2.5$ and to compute their 


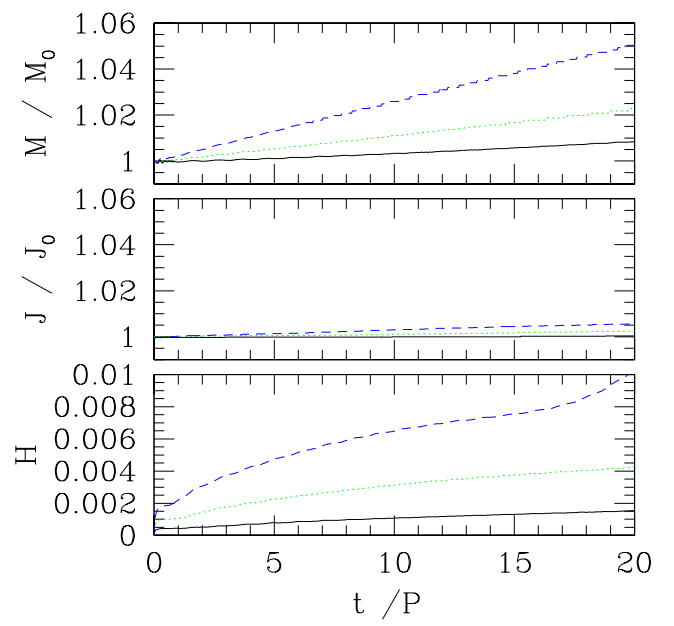

FIG. 2. Evolution of the ADM mass (top), angular momentum (middle), and violation of the Hamiltonian constraint (bottom) for $\Gamma=2.5$. Time is shown in units of the rotational period of the neutron stars. The dashed, dotted, and solid curves denote the results with $(91,91),(121,121)$, and $(181,181)$ grid resolutions, respectively.

fundamental oscillation frequency. Such simulation has not yet been accurately performed with HRSC schemes.

In Fig. 2, we show the evolution of the ADM mass, angular momentum, and the averaged violation of the Hamiltonian constraint (in which the baryon rest mass density is used for the weight; see [5] for definition) for $\Gamma=2.5$. (Similar results are obtained for $\Gamma=2$.) The figure shows that the conserved quantities remain conserved to high accuracy, particularly for the finest grid, and that the violation of the Hamiltonian constraint remains small. The outstanding feature is that the departure from angular momentum conservation with the HRC scheme is much smaller than with the HRSC scheme (see e.g. Fig. 5 in [13]). In the previous implementation the angular momentum gradually increases with time mainly due to the numerical error generated around the stellar surface and in the low-density atmosphere for which an artificial friction term was added to stabilize the computation. With the HRC scheme, such a drift in the angular momentum conservation is suppressed within $0.1 \%$ error after 20 rotation periods for grid resolutions with $N \geq 180$, probably due to less numerical inaccuracies around the stellar surface. These results indicate that the HRC scheme used is a robust scheme for the simulation of isolated neutron stars.

We now turn to present the results of numerical simulations of binary neutron star mergers. In the present test we choose binaries of equal mass with $1.3-1.3 M_{\odot}$ and $1.4-1.4 M_{\odot}$. As found in [6] using a HRSC scheme, a massive neutron star and a black hole are formed for the former and latter cases, respectively.

In Fig. 3, we show the time-evolution of the central value of the lapse function, $\alpha_{c}$, and the maximum value

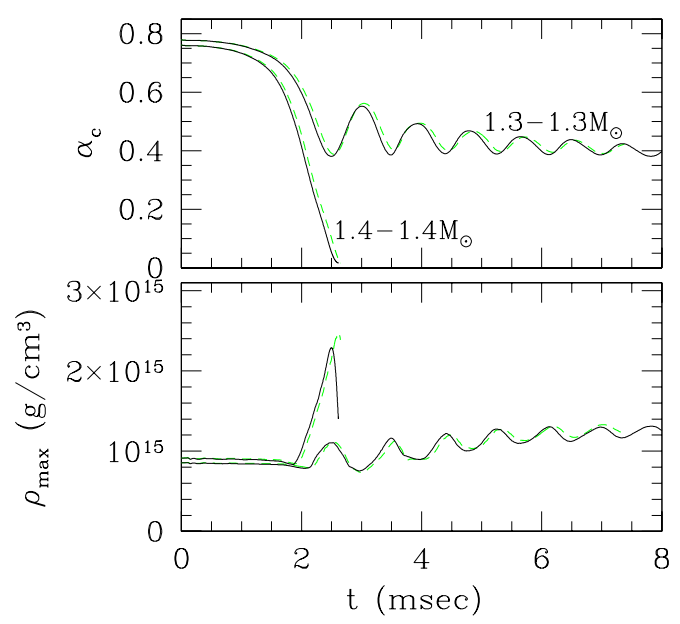

FIG. 3. Evolution of the maximum value of $\rho$ and the central value of $\alpha$ for the merger of equal-mass binary neutron stars, $1.3-1.3 M_{\odot}$ and $1.4-1.4 M_{\odot}$. In the former and latter cases, a massive neutron star and a black hole are the end-products of the merger, respectively.

of the density, $\rho_{\max }$, for these two models. The solid and dashed curves indicate the results obtained with the HRC and HRSC schemes, respectively. In the smaller mass case, a massive neutron star is formed after the merger, and hence, $\alpha_{c}$ and $\rho_{\max }$ show a series of small-amplitude oscillations until they eventually relax to quasi-stationary values. For both hydrodynamical schemes the amplitude and the frequency of the resulting neutron star oscillations agree well with each other. On the other hand, the outcome of the merger of the $1.4-1.4 M_{\odot}$ binary is a black hole, as can be directly inferred from the rapid collapse of the central lapse and the rapid growth of the maximum density (from $t \sim 2 \mathrm{~ms}$ onwards). Black hole formation is signaled by the appearance of an apparent horizon, which is detected in both implementations. In particular the time of formation of the apparent horizon agrees approximately for both schemes, with a time difference of about $0.07 \mathrm{~ms}$. For the two binary mergers considered, a small time lag in the evolution of $\alpha_{c}$ and $\rho_{\max }$ is observed between the two results computed by the different schemes. Its origin is likely the difference in the magnitude of the friction term around the stellar surface already discussed before which could generate an error in the angular momentum conservation. As mentioned above, this error is smaller with the HRC scheme.

In Fig. 4, we show the evolution of the ADM mass, angular momentum, and averaged violation of the Hamiltonian constraint for the $1.3-1.3 M_{\odot}$ binary merger. In this case, the ADM mass and angular momentum of the system show a gradual decrease due to the emission of gravitational waves [6]. Again, it is found that the two results agree well with each other within $\sim 0.5 \%$. The averaged violation of the Hamiltonian constraint remains approximately of identical magnitude, $\sim 0.02$, which in- 


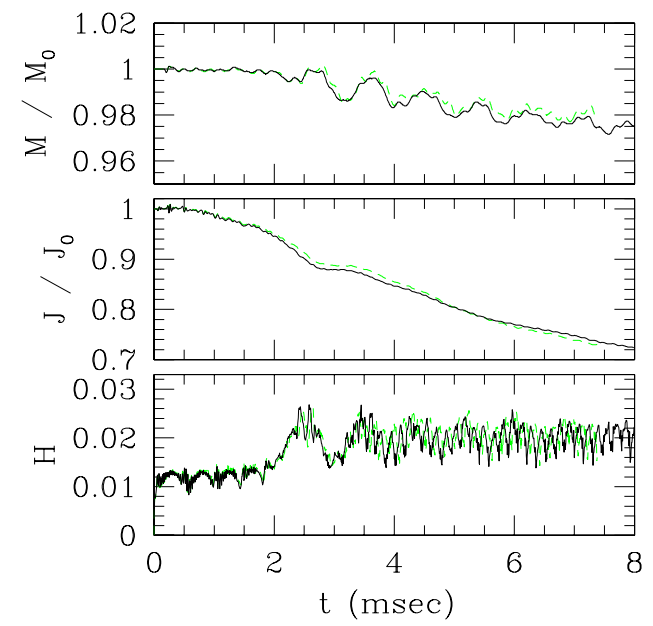

FIG. 4. Evolution of the ADM mass (top), angular momentum (middle), and violation of the Hamiltonian constraint (bottom) for the merger of binary neutron stars with masses $1.3-1.3 M_{\odot}$. The solid and dashed curves in all panels indicate the results obtained with the HRC scheme and with the HRSC scheme, respectively.

dicates that the accuracy of the results of the two hydrodynamical schemes is approximately identical.

To summarize, it has been shown through simulations of pulsating and rotating neutron stars, and binary neutron star mergers, that the results produced by the HRC scheme proposed by [12] agree well with those obtained with a Roe-type HRSC scheme. The accuracy measured by the evolution of the ADM mass, angular momentum, and violation of the Hamiltonian constraint in the HRC scheme are as good as or even better than those obtained for the HRSC scheme. In addition, the HRC scheme has a number of advantages to the HRSC scheme: (1) it is straightforward to implement since the solution of Riemann problems is avoided; hence one does not need to compute the complicated sets of eigenvectors of the Jacobian matrices associated with the fluxes (transport terms) of the hydrodynamics equations; (2) for this reason the computational costs of the HRC scheme are much less expensive, as the characteristic information required in HRSC schemes is not necessary. In the tests reported in this paper we have found that in our fully general relativisitc implementation, the computational time is saved by about $20 \%$; (3) the density of the unphysical atmosphere one needs to build around isolated stars when adopting the conservative form of the hydrodynamics equations can be several orders of magnitude smaller than that in HRSC schemes. Associated with this advantage the code can be applied for neutron stars with a large adiabatic index $\Gamma=2.5$.

These facts illustrate that HRC schemes can be useful and robust tools for hydrodynamical simulations in full GR involving dynamical spacetimes. In addition, their suitability over HRSC schemes becomes further appar- ent when the wave structure of the hyperbolic system to solve is unknown, as it is partially the case in general relativistic magnetohydrodynamics (GRMHD). Hence, HRC schemes can help the achievement of GRMHD simulations in which the equations to solve are more complicated than those of purely hydrodynamical flows [15].

Acknowledgments: The numerical simulations were performed on the FACOM VPP5000 computers at the data processing center of NAOJ. This work was in part supported by Monbukagakusho Grant (Nos. 15037204, 15740142 , and 16029202) and by the Spanish Ministerio de Ciencia y Tecnología (AYA2004-08067-C03-C01).

[1] A. Lucas-Serrano, J. A. Font, J. M. Ibánez, and J. M. Martí, Astron. Astrophys. 428, 703 (2004).

[2] M. Shibata, Phys. Rev. D 60, 104052 (1999).

[3] J. A. Font et al., Phys. Rev. D 65, 084024 (2002).

[4] M. Shibata and K. Uryū, Phys. Rev. D 61, 064001 (2000); Prog. Theor. Phys. 107, 265 (2002): M. Miller, P. Gressman, and W.-M. Suen, Phys. Rev. D 69, 064026 (2004): M. D. Duez, P. Marronetti, T. W. Baumgarte, and S. L. Shapiro, Phys. Rev. D 67, 024004 (2003): L. Baiotti et al., Phys. Rev. D 71, 024035 (2005).

[5] M. Shibata, K. Taniguchi, and K. Uryū, Phys. Rev. D 68, 084020 (2003).

[6] M. Shibata, K. Taniguchi, and K. Uryū, Phys. Rev. D 71, 084013 (2005).

[7] M. Shibata and T. Nakamura, Phys. Rev. D 52, 5428 (1995): T. W. Baumgarte and S. L. Shapiro, Phys. Rev. D 59, 024007 (1999).

[8] J. A. Font, Living Rev. Relativity 6, 4 (2003).

[9] J. M. Martí and E. Müller, Living Rev. Relativity 6, 7 (2003).

[10] L. Del Zanna and N. Bucciantini, Astron. Astrophys. 390, 1177 (2002): P. Anninos and P. C. Fragile, Astrophys. J. Suppl. 144, 243 (2003).

[11] E. F. Toro, Riemann Solvers and Numerical Methods for Fluid Dynamics (Springer Verlag, 1997).

[12] A. Kurganov and E. Tadmor, J. Comput. Phys. 160, 214 (2000).

[13] M. Shibata, Phys. Rev. D 67, 024033 (2003).

[14] P. Haensel and A. Y. Potekhin, Astron. Astrophys. to be published (astro-ph/0408324), and references therein.

[15] M. D. Duez, Y.-T. Liu, S. L. Shapiro, and B. Stephens, submitted to Phys. Rev. D; L. Antón et al, in preparation; M. Shibata and Y. Sekiguchi, in preparation. 